\title{
Detection of cirrus clouds by stereovision system
}

\author{
${ }^{1}$ Med Nadjib Kouahla, ${ }^{1}$ Brahim Farou, ${ }^{1}$ Zineddine Kouahla and ${ }^{1}$ Hamid Seridi \\ ${ }^{1}$ LabSTIC laboratory/University 8 may 1945 of Guelma Algeria
}

\begin{abstract}
The cirrus clouds is one of the objects existing in the troposphere how play an important role in the process of climate change. The research presented in this paper is aimed to present a model for detecting of clouds, presenting theirs distributions and giving a measure about altitudes by a stereovision system. Two cameras simultaneously take images of the same scene at two different observations sites. The principle consists to identify in both images matched pairs of points corresponding to physical points in the cloud. The observational program are conducted in France in June 2014.
\end{abstract}

Key words: Stereovision, detection, clouds.

\section{Introduction}

Stereovision system has proven a major contribution in several fields of application dedicated to 3D imaging in agricultural applications [1], industrial robot [2], for guidance of autonomous aircraft [3], aquaculture for measuring different fish features [4], and for object reconstruction like emissive layer [5] or cirrus clouds [6].

Cirrus clouds play an important role for climate change covering about $20 \%$ of the earth's surface, they constituted of ice particles is either natural, or artificial related to airplane exhausts [7]. The World Meteorological Organization has established a classification of three main types in the family of these clouds: cirrus, cirrocumulus, and cirrostratus [8].

Several methods have been developed to provide a system of detection and classification of clouds, either based on different instruments: ground based [9], positioned on meteorological balloons or satellite [10], other more complex models using numerous features and huge libraries as a training set.

In recent studies, some Classifiers are proposed to differentiate clouds by class, based upon the cloud characteristics (spectral, textural and physical). There is three groups: simple [9], statistical [11] and artificial intelligence classifiers [12].

In this paper, we propose a novel method based on stereovision system suitable for retrieving the altitude of a cloud because it is an indicator of the cloud type. This method generally use two views of the object of interest taken at the same time with two cameras or two views of the object in motion taken with a single camera [13] [14].

In section 2 we describe the stereoscopic system consisting of two cameras located in two sites separated by a distance $\mathrm{D}$. The optical axes have the same direction but are oriented face-to-face, the images are taken in principle simultaneously, but a maximum time difference of 30 seconds is allowed between the shots at the two sites. Section 3 is devoted to a description of the 
instrumentation and observations setup. In section 4 we present the triangulation software developed to calculate altitude of clouds. As the medium is a diffuse object, without any definite line or corner, we use a ZNCC parameter to identify matching pairs of points in both images. In the last part, some preliminary results obtained during the June 2014 campaign are presented.

\section{Stereoscopy Vision System}

Our stereoscopic vision system is composed of two real cameras oriented in vis-à-vis mode located in two different sites. This latter is completed by two virtual cameras vertically located in the observation sites at an altitude $\mathrm{L}$ that can be adjusted according to the height of the optical axis angle of the real cameras.

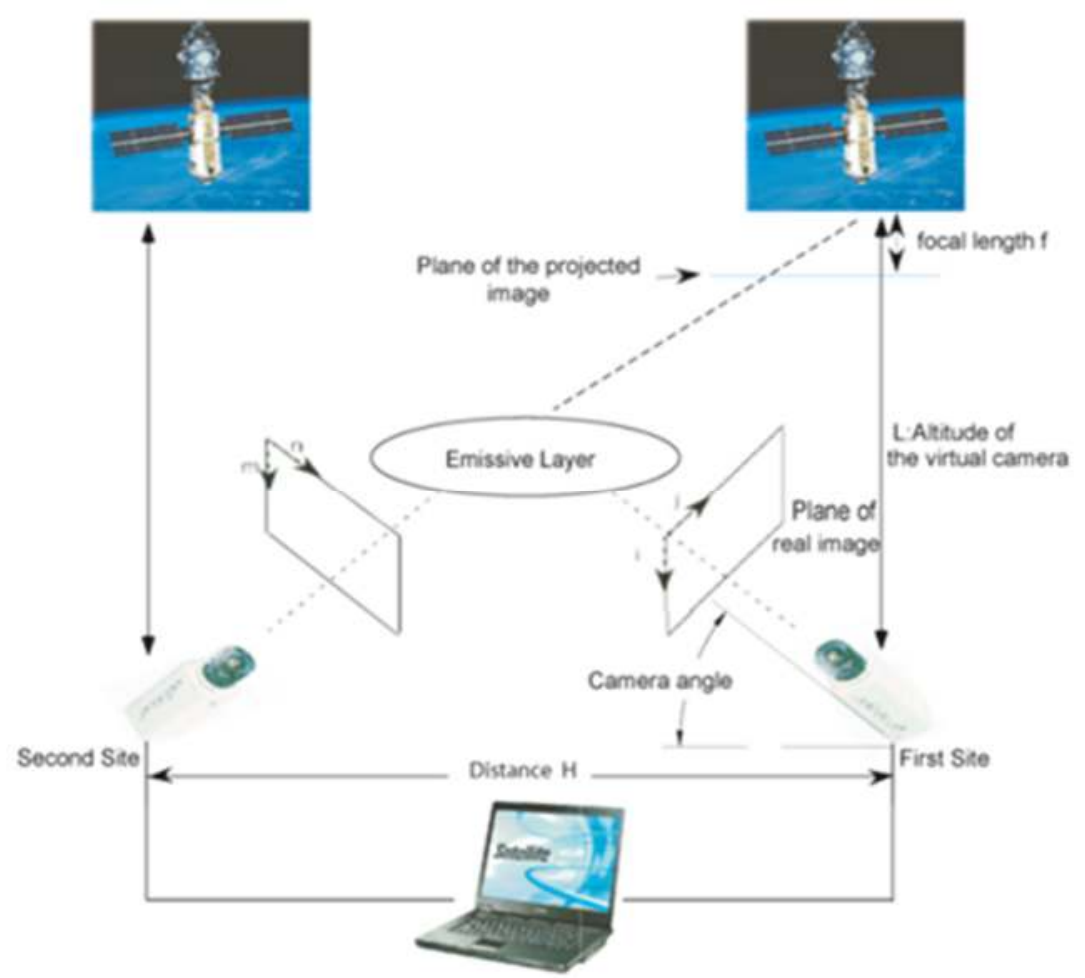

Figure1. Stereovision System.

Generally, when the height is set to $28^{\circ}$, we adopt $\mathrm{L}=250 \mathrm{Km}$ and the parameters of virtual cameras are assumed identical to the real ones. The two images taken simultaneously at two separate views are interconnected by epipolar geometry of the stereo system [15]. The problem of matching points between the two images will be easier when geometry is well defined (Figure 1).

The geometry of the system is summarized in two steps:

First, the "real" images are transformed into "virtual" images following a perspective reversal. Let us call the coordinates of the farthest away point present in the "real" image. 
The second step uses the principle of epipolar geometry [16]. The stereoscopic system is shown in Figure 2. The optical centers $\mathrm{C} 1$ and $\mathrm{C} 2$ of the virtual cameras are shown behind the images called (IM1) and (IM2) for clarity. Let us consider a point $\mathrm{N}$ in the layer. Its image point in (IM1) is called $\mathrm{m} 1$. The epipolar principle states that its matched point in (IM2) is located on a line called "epipolar segment" which is the intersection of planes (IM2) and $(\mathrm{C} 1 \mathrm{C} 2 \mathrm{~N})$. Now, we have to determine the location of $\mathrm{m} 2$ that is the matched point of $\mathrm{m} 1$.

Our purpose is to identify pairs of matched points in (IM1) and (IM2) and retrieve the position and altitude of the real geographic point $\mathrm{N}$. We first calculate the equation of the optical line $(\mathrm{C} 1 \mathrm{~m} 1)$ that joins the optical center of the camera $\mathrm{C} 1$ and the center of the current pixel $\mathrm{m} 1$ according to equation (1):

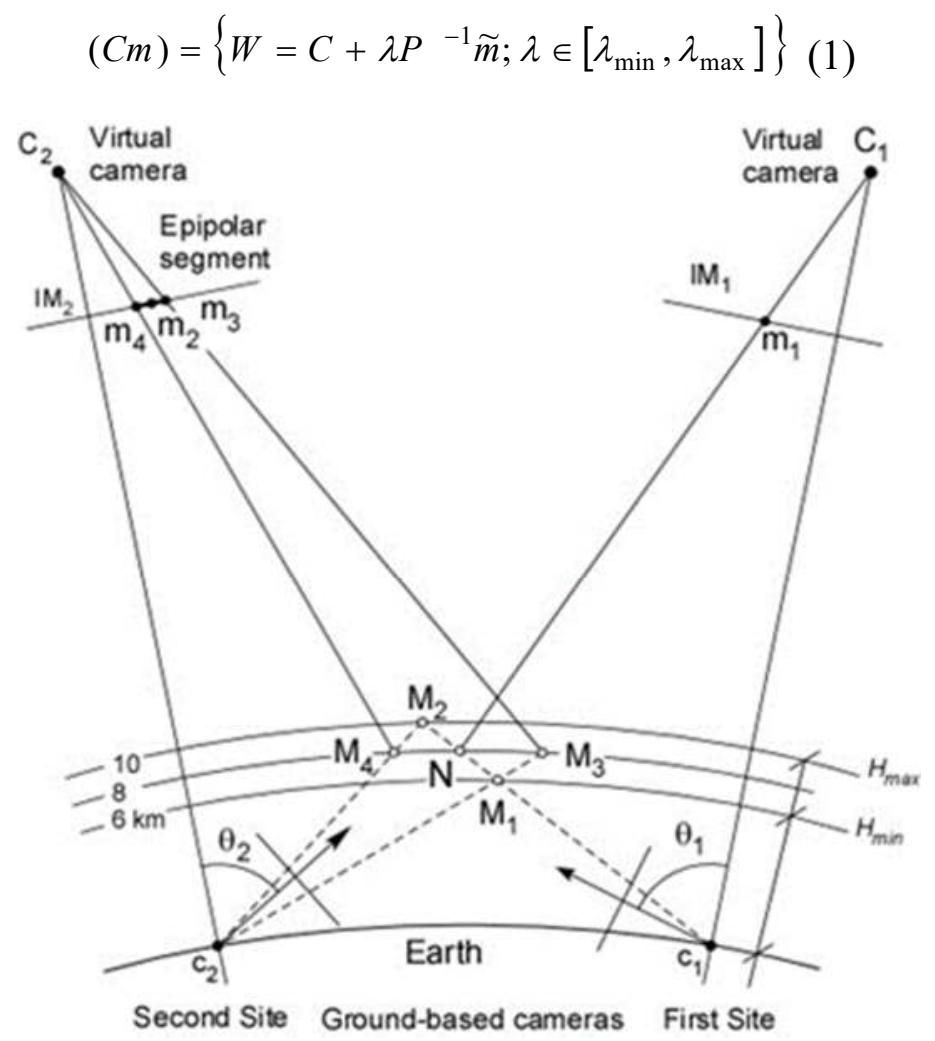

Figure 2. System Geometry.

\section{Observation sites and Instrumentation}

The observational program was conducted in France in June 2014 using two Canon cameras oriented NW-SE in a face-to-face mode. The first observation site was located at Marnay northwest of Besancon at an altitude of $275 \mathrm{~m}$, and the second site at Mont poupet southwest of Besancon at an altitude of $600 \mathrm{~m}$. The Cannon camera has 3888 × 2592 square CMOS pixels. The images are acquired using Lightview software and saved in JPEG format. Each camera is fined a computer driven alt-azimuthally astronomical platform (Figure 3). 


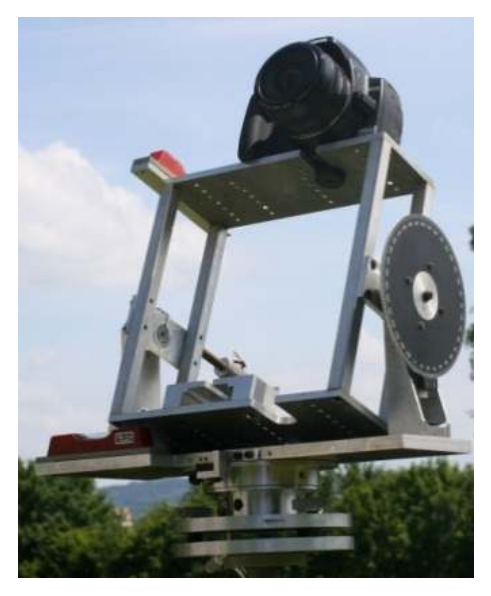

Figure 3. The observing instrument CANON EOS 400D.

\section{Results and discussion}

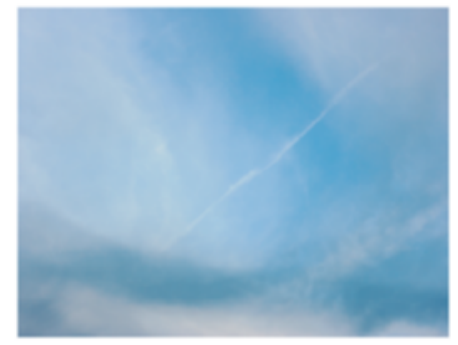

(a)

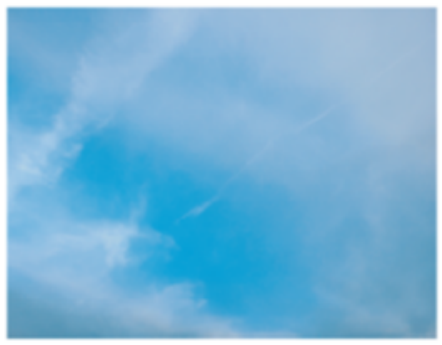

(b)

Figure 4. Pair of stereoscopic Image (a): Mont poupet, (b): Marnay

Figure $4(a, b)$ has a pair of image that shows the presence of wave fields whose intensity was measured. The two images will undergo treatments of conversion in Niveau de gris. This is followed by a reversal of perspective for getting a satellite type picture presenting a periodic structure Figure $4(\mathrm{a}, \mathrm{b})$.

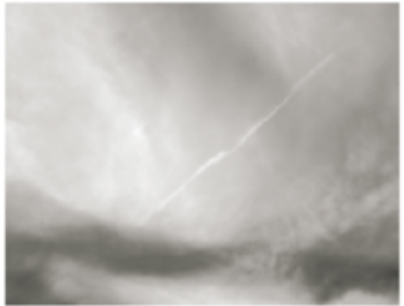

(a)

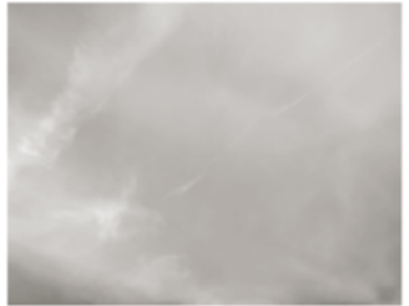

(b) 

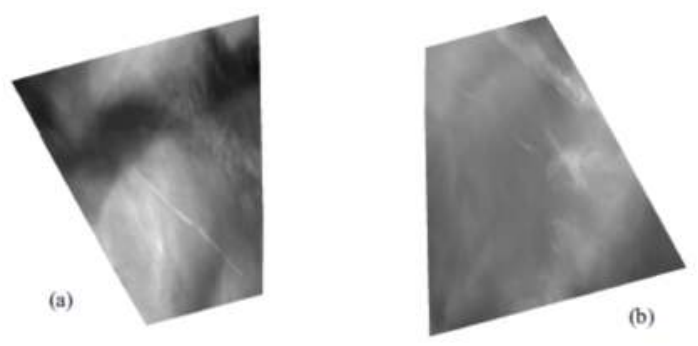

Figure 5. Projection-type satellite.

The images are superposed for extracting the common stereoscopic area and determining the pairs of points Figure 5 (c, d). The object is a gaseous layer without contour. To restore the altitude, triangulation software is employed for computing a correlation coefficient at every point.

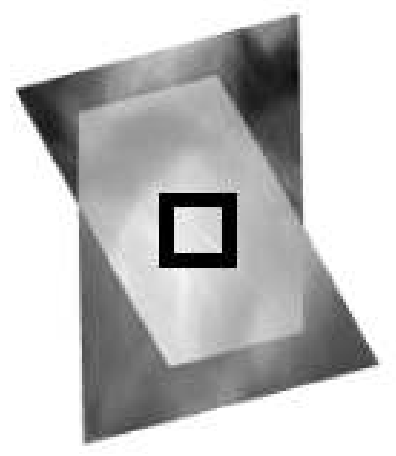

Figure 6. Superposition of the projected images (5.c) and (5.d).

The altitude measurement on regions of interest projections of the clouds are made. The following figure shows an example calculation of pairs of points on a region of size 100 pixels. 

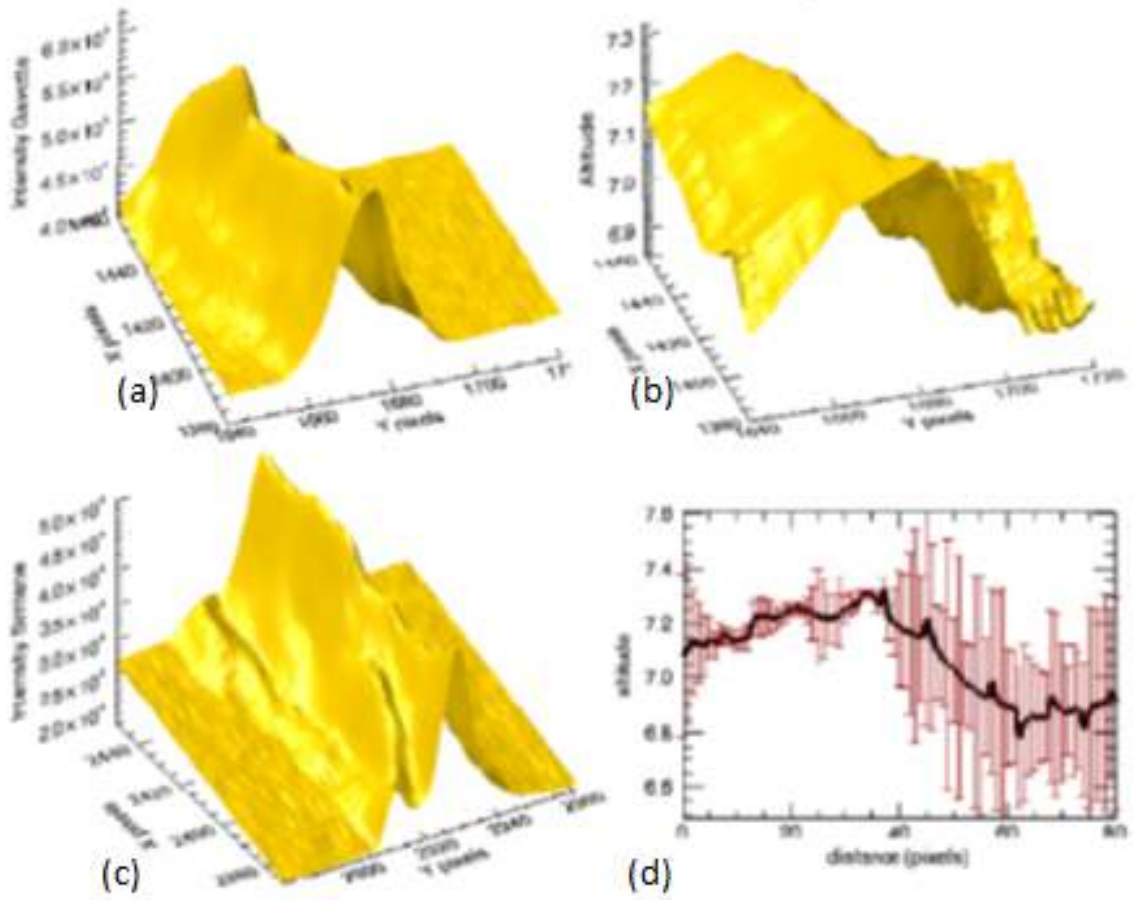

Figure 7. Triangulation algorithm results.

The figure 7 clearly shows that there is an analogy between the structures present in the altitude map of the layer and the maps of the intensities measured from the two observation sites. These maps correspond to regions of 100 pixels a side; the size of the regions has been chosen to cover at least one ripple of the emission and the elementary area on which we compute a cross correlation parameter (typically $5 * 5$ pixels) is small in size relatively to the wavelength of the oscillations. To avoid edge effects, the maps have been performed in the area where the calculation of the parameter ZNCC is not trimmed at the edges. Indeed, the map of an area of 100 pixels is reduced to a square of $80 * 80$ pixels [5]. Finally, we can propose to use the barycenter altitude of area as a parameter of a classification clouds process.

\section{Conclusion}

Our system is well adapted for detection clouds in the upper troposphere as well as cirrus. The joint use of stereoscopic, inversion projection and image processing on images obtained from observation campaign in France have given good results. The study presented in this paper aims to show the utility of stereovision system give the best results in average altitude used as a parameter of clouds detection.

\section{REFERENCES}

[1] V. Arellano, M., Griepentrog, H. W., Reiser, D., \& Paraforos, D. S. (2016). 3-D imaging systems for agricultural applications - a review. Sensors, 16(5), 618.

[2] Švaco, M., Šekoranja, B., Šuligoj, F., \& Jerbić, B. (2014). Calibration of an industrial robot using a stereo vision system. Procedia Engineering, 69, 459-463.

[3] Moore RJD, Thurrowgood S, Soccol D, Bland D, Srinivasan MV (2011) A bio-inspired stereo vision system for guidance of autonomous aircraft. In AsimBhatti (Ed.) Advances in theory and 
applications of stereo vision. Rijeka, Croatia: InTech. pp. 305-326.

[4] Saberioon, M., Gholizadeh, A., Cisar, P., Pautsina, A., \& Urban, J. (2017). Application of machine vision systems in aquaculture with emphasis on fish: state-of-the-art and key issues. Reviews in Aquaculture, 9(4), 369-387

[5] Moreels, G., Clairemidi, J., Faivre, M., Mougin-Sisini, D., Kouahla, M. N., Meriwether, \& Veliz, O. (2008). Stereoscopic imaging of the hydroxyl emissive layer at low latitudes. Planetary and Space Science, 56(11), 1467-1479.

[6] Kouahla, M. N., Faivre, M., Moreels, G., \& Seridi, H. (2016). 3D reconstruction of tropospheric cirrus clouds. Advances in Space Research, 58(7), 1362-1375.

[7] Dowling, D.R., Radke, L.F., 1990. A summary of the physical properties of cirrus clouds. J. Appl. Meteorol. 29, 970-978.

[8] International Cloud Atlas, part I, World Meteorological Organization, 1975, $183 \mathrm{p}$

[9] Heinle, A., Macke, A., Srivastav, A., 2010. Automatic cloud classification of whole sky images. Atmospheric Measurement Techniques 3, 557-567.

[10] Ricciardelli, E., Romano, F., Cuomo, V., 2010. A technique for classifying uncertain MOD35/MYD35 pixels through Meteosat second generation spinning enhanced visible and infrared imager observations. IEEE Transactions on Geoscience and Remote Sensing 48, 21372149.

[11] Ameur, Z., Ameur, S., Adane, A., Sauvageot, H., Bara, K., 2004. Cloud classification using the textural features of Meteosat images. International Journal of Remote Sensing 25, 4491-4503. [12] Ma, Y., Soatto, J.,Kosecka, and S. S. Sastry, An Invitation to 3-D Vision, From Images to Geometric Models, 526 pp., 2004

[13] S.K. Park, I.S. Kweon, "Robust and direct estimation of 3-d motion and scene depth from stereo image sequences," Pattern Recognition. 32, 1713-1728, 2001

[14] O. Faugeras, G. Toscani, The calibration problem for stereo, In IEEE, Proceedings of the International Conference on Computer Vision and Pattern Recognition., Miami Beach, 1986, pp. 15-20.

[15] D. Pautet, G. Moreels. "Ground-based satellite-type images of the upper atmosphere emissive layer”, Applied Optics, 41, (2002) 823-831

[16] Hartley, R., Zisserman, A.: Multiple View Geometry in Computer Vision. Cambridge university Press, Cambridge, 2003 